\title{
Yak-milk-derived exosomes promote proliferation of intestinal epithelial cells in an hypoxic environment
}

\author{
H. N. Gao, ${ }^{1,2}$ H. Y. Guo, ${ }^{2,3}$ H. Zhang, ${ }^{2,3}$ X. L. Xie, ${ }^{4}$ P. C. Wen, ${ }^{1 *}$ and F. Z. Ren ${ }^{1,2,3 *}$ \\ ${ }^{1}$ College of Food Science and Engineering, Gansu Agricultural University, Lanzhou 730070, China \\ ${ }^{2}$ Key Laboratory of Functional Dairy, College of Food Science and Nutritional Engineering, China Agricultural University, Beijing, 100083, China \\ ${ }^{3}$ Beijing Advanced Innovation Center for Food Nutrition and Human Health, College of Food Science and Nutritional Engineering, \\ China Agricultural University, Beijing, 100094, China \\ ${ }^{4}$ Treasure of Tibet Yak Dairy Co., Ltd., Lhasa, 610000, China
}

\section{ABSTRACT}

Intestinal epithelial cells (IEC) are an important part of the intestinal barrier. Barrier function was disrupted under hypoxia, but milk-derived exosomes can regulate the intestinal barrier function. However, the mechanisms underlying the association between yak milk exosomes and hypoxia in IEC remain poorly understood. In this follow-up study, we proposed an effective optimization method for purifying yak-milkderived exosomes. The Western blot analyses indicated that the expression of the proteins of the endosomal sorting complexes required for transport (TSG101), proteins of the tetraspanin family (CD63), and heat shock protein 70 (Hsp-70) proteins from yak-milkderived exosomes were significantly higher than those in cow-milk-derived exosomes. Flow cytometry analysis showed that yak milk had 3.7 times the number of exosomes compared with cow milk. Moreover, we explored whether yak milk exosomes could facilitate intestinal cell survival under hypoxic conditions in vitro. The 3-(4,5-dimethylthiazol-2-yl)-2,5-diphenyl tetrazolium bromide results showed that yak-milk-derived exosomes significantly increased survival of IEC-6 cells with rates of up to $29 \%$ for cells incubated in hypoxic conditions for $12 \mathrm{~h}$, compared with those of cow-milk-derived exosomes posttreatment (rates of up to $22 \%$ for cells incubated in hypoxic conditions for $12 \mathrm{~h}$ ). Confocal microscopy revealed that the IEC-6 cells uptake more yak-milk-derived exosomes than cow milk in hypoxic conditions. Furthermore, the Western blot analyses indicated that yak-milk-derived exosomes significantly promote oxygen-sensitive prolyl hydroxylase (PHD)-1 expression and decrease the expression of hypoxiainducible factor- $\alpha$ and its downstream target vascular endothelial growth factor (VEGF) in the IEC-6 cells.

Received April 19, 2018.

Accepted October 2, 2018.

*Corresponding authors: wenpch@126.com and renfazheng@263.net
Further, yak-milk-derived exosomes significantly inhibited p53 levels. In conclusion, our findings demonstrate that yak-milk-derived exosomes more effectively activate the hypoxia-inducible factor signaling pathway, thus promoting IEC-6 cell survival, which may result in higher hypoxia tolerance than cow-milk-derived exosomes.

Key words: milk exosome, yak, intestinal epithelial-6 cell, hypoxia

\section{INTRODUCTION}

Changes in environmental conditions have produced diversification of animal species, resulting in large differences in the nutritional components of milk from different mammals, which can provide nutritional benefits to humans (Yang et al., 2014). Yaks live on the Qinghai-Tibet Plateau, at altitudes of 2,500 to 6,000 $m$ (Nivsarkar et al., 1997). These harsh environmental conditions have resulted in the key adaptations of hypoxia resistance and enhanced metabolic capacity (Qiu et al., 2012).

The intestinal epithelial cells (IEC) of the gastrointestinal tract (GIT) play a crucial role in the regulation of development and health, forming an essential barrier to the exterior of the body, and are responsible for the first physiological step of transporting nutrients to the body's cells. Both the absorption and barrier functions of the intestinal epithelium are regulated by the availability of $\mathrm{O}_{2}$ (Taylor and Colgan, 2007). Previous studies have shown that hypoxia triggers the loss of the epithelial barrier as well as mucosal inflammatory disease, such as inflammatory bowel disease (Karhausen et al., 2004; Colgan and Taylor, 2010). Ongoing studies suggest that IEC cells induce hypoxia-regulated responses, including hypoxia-inducible factors (HIF) and vascular endothelial growth factor (VEGF), in hypoxic environments (Aro et al., 2012; Jiang et al., 2016; Sun et al., 2017). Both pathways are tightly regulated by oxygen-sensitive prolyl hydroxylases 
(PHD), which represent promising therapeutic targets for mucosal inflammatory disease (Colgan and Taylor, 2010). Hypoxia-inducible factor $1 \alpha(\mathbf{H I F}-1 \boldsymbol{\alpha})$ plays an important role in cell survival and regeneration in response to decreased oxygen availability (Jiang et al., 2016; Brown and Taylor, 2018).

Recent studies have reported a role of exosomes in signaling, and exosomes can carry complex biological information, in the form of mRNA and microRNA, in the crosstalk between various cell types (Belting and Wittrup, 2008; Al-Nedawi et al., 2009; Cocucci et al., 2009; Théry et al., 2009). Exosomes are nano-sized endosome-derived membrane vesicles $(50-200 \mathrm{~nm}$ in diameter; Li et al., 2016), and their density ranges from 1.13 to $1.19 \mathrm{~g} / \mathrm{mL}$ (Théry et al., 2006). In recent years, exosomes have been identified in human (Kosaka et al., 2010; Zhou et al., 2012; Munch et al., 2013), cow (Chen et al., 2010; Hata et al., 2010; Gu et al., 2012; Izumi et al., 2012; Sun et al., 2013; Izumi et al., 2015), and pig (Gu et al., 2012) milk. Humans absorb milk-derived exosomes and deliver low-molecular-weight metabolites that are encapsulated in milk-derived exosomes to peripheral tissues (Chen et al., 2010; Wolf et al., 2015; Benmoussa et al., 2016; Liao et al., 2017). It has been suggested that milk-derived exosomes may pass the intestinal barrier and reach the systemic circulation, where they can facilitate intestinal cell proliferation and intestinal tract development (Chen et al., 2016; Hock et al., 2017).

Thus, we hypothesized that yak-milk-derived exosomes can regulate IEC-6 cell proliferation and intestinal tract development in high altitude hypoxia through hypoxia-related pathways. We hope this study will provide new evidence for the regulation of intestinal tract development by yak-milk-derived exosomes in humans.

\section{MATERIALS AND METHODS}

\section{Experiment 1: Characterization of Exosomes in Yak and Cow Milk}

Milk Sample Preparation. Twenty-seven healthy Chinese Holstein cows in mid-lactation period (145 \pm 20 d postpartum; Bos taurus) milk samples were collected from local dairy farms in Beijing, and 27 healthy yaks (Bos grunniens) in mid-lactation period $(145 \pm 20$ d postpartum) milk samples were collected from a farm in Tibet and a farm in Gansu province, respectively. Raw milk samples from each farm were pooled into 3 fractions. The use and care of the animals used in Procedures involving animals were approved by the Animal Care Committee of the China Agricultural University (ID: SKLAB-B-2010-003)The milk samples were stored at $-20^{\circ} \mathrm{C}$ immediately after collection and were later transferred to our laboratory and stored at $-80^{\circ} \mathrm{C}$ until use.

Exosome Isolation. Bovine and yak milk exosomes were isolated according to the modified literature (Wolf et al., 2015; Zaborowski et al., 2015; Benmoussa et al., 2016; Kusuma et al., 2016; Yang et al., 2017). Method 1 (ultracentrifugation) whole-milk samples were centrifuged $\left(8,000 \times g, 4^{\circ} \mathrm{C}, 30 \mathrm{~min}\right)$ to remove fat, cells, and large debris. The defatted supernatant was then centrifuged $\left(13,200 \times g, 4^{\circ} \mathrm{C}, 1 \mathrm{~h}\right)$ to remove residual fat and cell debris. The whey supernatant was then ultracentrifuged $\left(120,000 \times g, 4^{\circ} \mathrm{C}, 90 \mathrm{~min}\right)$ using an SW41T rotor (Beckman Coulter Instruments, Fullerton, CA). Finally, the exosomes were successively passed through $0.45-$ and $0.22-\mu \mathrm{m}$ filters and then resuspended in PBS (catalog no. C0221A, Beyotime Institute of Biotechnology, Shanghai, China). Method 2 (ultracentrifugation with rennet precipitation) whole-milk samples were centrifuged $\left(8,000 \times g, 4^{\circ} \mathrm{C}, 30 \mathrm{~min}\right)$ to remove fat, cells, and large debris. The defatted supernatant was then centrifuged $\left(13,200 \times g, 4^{\circ} \mathrm{C}, 1 \mathrm{~h}\right)$ to remove residual fat and cell debris. Rennet (0.025 g/L; Naturen Stamix 1150 NB, Chr. Hansen, Hoersholm, Denmark) was added to the defatted supernatant, which was then incubated at $37^{\circ} \mathrm{C}$ for $6 \mathrm{~h}$ to remove casein (Zhang et al., 2017). The clear supernatant (whey) was then passed through a $0.45-\mu \mathrm{m}$ filters to remove residual cell debris and ultra-centrifuged $\left(120,000 \times g, 4^{\circ} \mathrm{C}, 90 \mathrm{~min}\right)$ using an SW41T rotor. The pelleted exosomes were resuspended in PBS and then ultra-centrifuged $(120,000$ $\left.\times g, 4^{\circ} \mathrm{C}, 90 \mathrm{~min}\right)$ for washing. Finally, the exosomes were successively passed through 0.45 - and $0.22-\mu \mathrm{m}$ filters, and then resuspended in PBS. The protein content of the exosomes was determined using bicinchoninic acid assay kits (catalog no. P0010S, Beyotime Institute of Biotechnology).

Transmission Electron Microscopy. The exosomes in deionized water were placed on a 300-mesh nickel grid and allowed to dry for $30 \mathrm{~min}$. The excess solution was removed using a filter paper, and the grid was stained with $2 \%$ uranyl acetate in water for 10 min, and washed by transferring it onto several drops of deionized water and incubated overnight and further drying. The grid was then analyzed using a JEOL JEM1200EX Transmission Electron Microscope (JEOL, Tokyo, Japan).

Exosome Size Analysis. The particle size distribution and zeta potential were determined with a Zeta-sizer Nano-series instrument (Malvern Nano-ZS, Malvern, UK) at $4^{\circ} \mathrm{C}$. The exosomes were diluted 1,000 times with ultrapure water. The size data refer to the scattering intensity distribution.

Western Blot Analysis. The samples (yak and cow exosomes) were lysed using ice-cold radio-immuno- 
precipitation assay buffer (catalog no. P0013, Beyotime Institute of Biotechnology) containing $1 \mathrm{~m} M$ phenylmethylsulfonyl fluoride and $1 \%$ ( $\mathrm{vol} / \mathrm{vol}$ ) of protease and phosphatase inhibitor cocktail (catalog no. 78430, Thermo Scientific, Rockford, IL). The lysates $(25 \mu \mathrm{g}$ of protein) were loaded onto gels, separated using $10 \%$ SDS-PAGE, and blotted onto polyvinylidene fluoride transfer membranes (0.45 and $0.2 \mu M$ immobilon-P, catalog no. IPVH00010 and ISEQ00010, Millipore, Billerica, MA). The membranes were blocked with $5 \%$ skim milk-TBS-Tween 20 for $4 \mathrm{~h}$ at room temperature (RT) and incubated overnight at $4^{\circ} \mathrm{C}$ with the primary antibodies (diluted 1:1,000 in PBS) against proteins of the tetraspanin family (CD63; catalog no. MCA2041GA, Bio-Rad, Hercules, CA), heat shock protein-70 (Hsp70; catalog no. ab2787, Abcam, Cambridge, UK), Calnexin (catalog no. NB100-1965, Novus Biologicals Europe, Abingdon, UK), proteins of the endosomal sorting complexes required for transport (TSG101; catalog no. AV38773, Sigma, St. Louis, MO; Théry et al., 2006; Lässer et al., 2011; Benmoussa et al., 2016; Chen et al., 2016), and $\beta$-actin (catalog no. abs132184, 1:500, Absin, Absin Bioscience Co. Ltd., Shanghai, China). The blots were incubated with the secondary antibody (anti-rabbit IgG, catalog no. A9169, Sigma; anti-mouse IgG, catalog no. A9044, Sigma) diluted at 1:3,000 in PBS.

The immunoreactive bands were visualized using the Pierce ECL Western Blot Substrate (catalog no. 32106, Thermo Scientific, Rockford, IL) according to the manufacturer's instructions and exposed by Image Quant LAS4000 (GE Healthcare, Little Chalfont, UK). The protein band densities were assayed with the Image J2x 2.1.4.7 Analyzer (Rawak Software Inc., Stuttgart, Germany). Band densities of exosome-supplementation treatments were normalized to that of $\beta$-actin.

Exosome Characterization and Quantification Using Flow Cytometry. The exosomes were characterized and quantified using a CD63 intensity plot. The ratio of labeled with CD63 to exosomes determined the characterization and concentration of the milk exosomes per milliliter. The exosomes were fixed with ice-cold methanol for 15 min. Nonspecific reactivity was blocked with 1\% BSA (catalog no. ST023, Beyotime Institute of Biotechnology) incubated overnight at $4^{\circ} \mathrm{C}$ and incubated with the primary antibodies (diluted 1:100 in 1\% BSA) against CD63 (catalog no. MCA2041GA, BioRad, Hercules, CA) for 6 h. After washing 3 times with PBS, the exosomes were incubated with the secondary antibody Alexa Fluor 488 (Anti-Mouse IgG, catalog no. 4408, Cell Signaling, Danvers, MA) diluted at 1:1,000 in PBS for $2 \mathrm{~h}$ at RT, washed, and analyzed with flow cytometry. Acquisition was performed using the Image
Stream-X Imaging Flow Cytometer (Amnis Corporation, Seattle, WA) equipped with INSPIRE software. A minimum of 100,000 exosomes were analyzed for each sample. Data analysis was performed using the IDEAS software (Amnis Corporation).

\section{Experiment 2: Yak- and Cow-Milk-Derived Exosomes Induce IEC-6 Cell Proliferation in Hypoxia}

Cell Culture and Treatment with Yak- and Cow-Milk-Derived Exosomes. The IEC-6 cells were obtained from iCell (Bioscience Inc., Shanghai, China). The cells were cultured in $3.5-\mathrm{cm}$ dishes (cata$\log$ no. 172958 , Thermo Scientific) incubated at $37^{\circ} \mathrm{C}$ containing 5\% $\mathrm{CO}_{2}$ (normoxic). Dulbecco's modified Eagle medium (DMEM)/Ham's F-12 (diluted 1:1, catalog no. 1439945/1491066, Invitrogen Trading Co. Ltd., Shanghai, China) containing $10 \%$ fetal cow serum (Invitrogen Trading Co. Ltd.) and $100 \mu \mathrm{g} / \mathrm{mL}$ of Penicillin-Streptomycin Solution (catalog no. C0222, Beyotime Institute of Biotechnology) were used as the basal growth medium until $80 \%$ confluence was reached. The cells were then passaged with $0.25 \%$ trypsin- $0.02 \%$ EDTA (catalog no. C0203, Beyotime Institute of Biotechnology).

3-(4,5-Dimethylthiazol-2-yl)-2,5-Diphenyl Tetrazolium Bromide Assay. Cells were seeded in 96-well microplates by culturing a suspension of 5,000 cells $(100 \mu \mathrm{L} /$ well $)$ in DMEM/F-12 containing $10 \%$ fetal cow serum for $24 \mathrm{~h}$. Afterward, the cells were serum starved overnight and then cultured in $200 \mu \mathrm{L}$ of DMEM with varying concentrations of cow- and yak-milk-derived exosomes (0, 40, 80, 120, 160, 200, and $240 \mathrm{ng}$ of protein $/ \mu \mathrm{L})$, and then transferred into an atmosphere with $5 \% \mathrm{CO}_{2}$ (normoxic) and $5 \% \mathrm{CO}_{2}$, $95 \% \mathrm{~N}_{2}$ (hypoxic; Qian Xi, Beijing, China) for 12, 24, and $48 \mathrm{~h}$, respectively. Each concentration was tested in 6 independent wells, and the experiment was considered to have a completely randomized design. After the indicated time, the DMEM was removed, and $100 \mu \mathrm{L}$ of $0.5 \mathrm{mg} / \mathrm{mL} \mathrm{3-(4,5-dimethylthiazol-2-yl)-2,5-diphenyl}$ tetrazolium bromide (MTT; Amresco, Solon, $\mathrm{OH}$ ) was added, and the samples were then incubated for $4 \mathrm{~h}$. The formazan crystals were solubilized in $150 \mu \mathrm{L}$ of dimethyl sulfoxide (catalog no. D 4045-1L, Sigma), and the plates were gently shaken for 3 min each to achieve complete dissolution. The absorbance was measured at $450 \mathrm{~nm}$ using an automatic ELISA reader. The results are presented as the ratio of the relative viability rate of the yak- and cow-milk-derived exosome-treated wells in hypoxic relative to positive control. Positive control, IEC-6 cells in normoxia conditions (absence of yak- and cow-milk-derived exosomes). 
Experiment 3: Uptake of Yak- and Cow-Milk-Derived Exosomes by IEC-6 Cells in Hypoxic Staining of Exosomes

Purified exosomes were labeled with a yellow-orange fluorescent dye with long aliphatic tails (PKH26, catalog no. P9691, Sigma) according to the manufacturer's protocol, with some modifications. Exosome pellets were resuspended in $1 \mathrm{~mL}$ of Diluent $\mathrm{C}$ (at a concentration of $240 \mathrm{ng}$ of exosomes $/ \mu \mathrm{L})$. Separately, $4 \mu \mathrm{L}$ of PKH26 was mixed with $1 \mathrm{~mL}$ of Diluent C. The exosome resuspension solution was mixed with the PKH26 working solution and incubated for $10 \mathrm{~min}$. The labeling reaction was stopped by adding an equal volume of $1 \%$ BSA and washed with PBS 3 times. The same concentration of PKH26 was centrifuged in parallel to create a background control for possible pelleted unbound dye.

The IEC-6 cells were incubated with PKH26-labeled exosomes working solution (at a concentration of $240 \mathrm{ng}$ of exosomes $/ \mu \mathrm{L}$ ) or the same volume of the PKH26-PBS control, and they were then incubated serum-free for 12 $\mathrm{h}$ at $37^{\circ} \mathrm{C}$. After incubation, the cells were washed with PBS 3 times, fixed with $4 \%$ paraformaldehyde working solution for $30 \mathrm{~min}$, and washed with PBS 3 times, stained with 4',6'-diaminido-2-phenylindole (C1002, Beyotime Institute of Biotechnology) working solution for $15 \mathrm{~min}$ at RT and washed with PBS 3 times. Cellular uptake of labeled exosomes was determined and analyzed using fluorescent and confocal microscopy (FV10-MCPSU, Olympus, Tokyo, Japan).

\section{Experiment 4: Effect on HIF- and p53-Related Protein} Expression by IEC-6 Cells in Hypoxia

Cell Culture and Treatment with Yak- and Cow-Milk-Derived Exosomes. When the seeded IEC-6 cells reached $80 \%$ confluency, they were treated with $240 \mathrm{ng} / \mu \mathrm{L}$ of yak- and cow-milk-derived exosomes, then transferred into an atmosphere with $5 \%$ $\mathrm{CO}_{2}$ (normoxic) and $5 \% \mathrm{CO}_{2}, 95 \% \mathrm{~N}_{2}$ (hypoxic; Qian $\mathrm{Xi}$, Beijing, China) for $12 \mathrm{~h}$.

Western Blot Analysis. The samples (IEC-6 cells) were lysed and analyzed with Western blotting according to the protocol used for yak exosomes as previously mentioned (experiment 1). The membranes were incubated overnight at $4^{\circ} \mathrm{C}$ with the primary antibodies

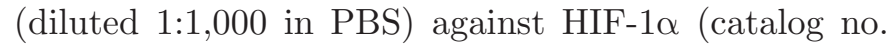
abs120168, Absin), PHD-1 (catalog no. abs125151a, Absin), VEGF (catalog no. abs123646, Absin), p53 (catalog no. 2524, Cell Signaling), and $\beta$-actin (catalog no. abs132184, Absin; 1:500). The blots were incubated with the secondary antibody (anti-rabbit IgG, catalog no. A9169, Sigma; anti-mouse IgG, catalog no. A9044,
Sigma) diluted at 1:3,000 in PBS. The immunoreactive bands were visualized using the Pierce ECL Western Blot Substrate as previously mentioned (experiment 1). Band densities of exosome-supplementation treatments were normalized to $\beta$-actin.

\section{Statistical Analysis}

For all experiments, the treatment means were compared using Tukey's test for post-hoc multiple comparisons of treatment means. Differences between experimental groups were determined using the Tukey's test for post-hoc multiple comparisons of treatment means and the random effect in the one-way ANOVA model with a post-hoc test of least significant difference using SAS software (version 9.2, SAS Institute Inc., Cary, NC). Differences were considered statistically significant at $P<0.05$. Results were presented as mean \pm standard error of the mean and represent independent biological replicates.

\section{RESULTS}

\section{Experiment 1: Characterization of Exosomes from Yak and Cow Milk}

We isolated yak-milk-derived exosomes using a differential method (Supplemental Figures S1 and S2; https: //doi.org/10.3168/jds.2018-14946). Transmission electron microscopy (TEM) showed that the yak-milk-derived exosomes were 50- to 200-nm vesicles surrounded by a cup-shaped lipid layer (Supplemental Figure S1A; https://doi.org/10.3168/jds.2018-14946). Nanoparticle size distribution analysis showed a peaking diameter of $109.2 \pm 57.1 \mathrm{~nm}$ with a polydispersity index of 0.256 by isolation method 1 compared with a peaking diameter of $112.4 \pm 48.6 \mathrm{~nm}$ with a polydispersity index of 0.167 by isolation method 2 (Supplemental Figure S1B; https://doi.org/10.3168/jds.2018-14946). The results of the nanoparticle size distribution analysis were consistent with the TEM results. The presence of the known exosome markers TSG101, CD63, and Hsp70, and the absence of endoplasmic reticulum (ER) marker calnexin were analyzed by Western blot (Supplemental Figures S1C and S1D; https://doi.org/10.3168/jds.2018 -14946). Method 2 resulted in slightly higher expression of the exosome-specific surface markers than method 1. We then confirmed the exosome count, which was analyzed by flow cytometry (Supplemental Figure S2C; https://doi.org/10.3168/jds.2018-14946). Compared with method 1, method 2 showed a higher exosome number (3 times compared with method $1 ; P<0.001$ ). Our results showed that method 2 is more suitable than 
A
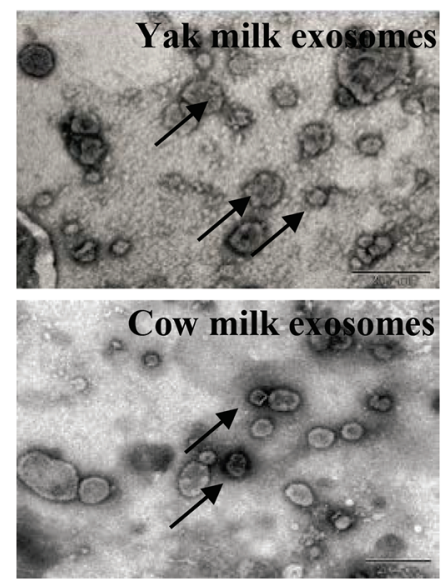

$\mathbf{C}$

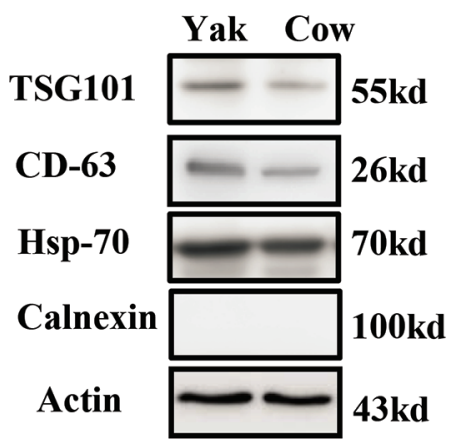

B
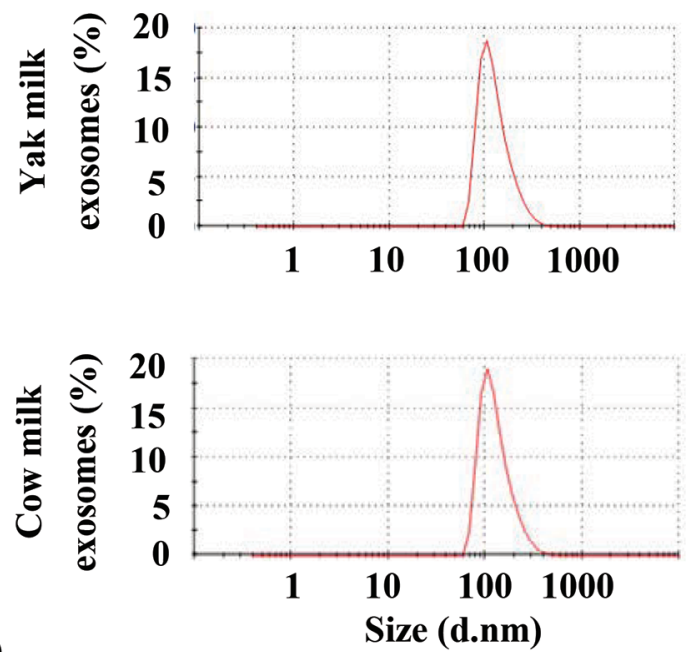

D

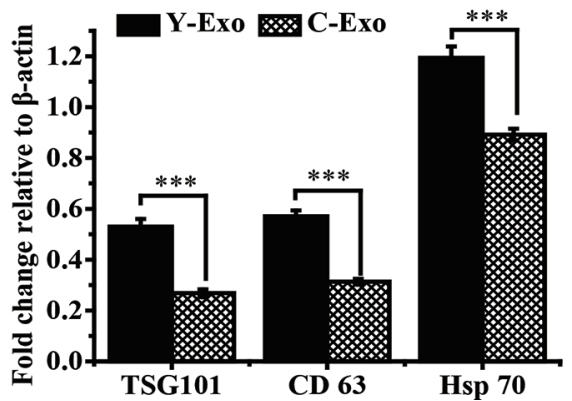

Figure 1. Characterization of optimized milk-derived exosomes. (A) Transmission electron microscopy images showing yak-milk-derived exosomes (upper) and cow-milk-derived exosomes (lower; scale bar: $200 \mathrm{~nm}$ ). Arrows indicate exosomes. (B) The size distributions of yak-milkderived exosomes (upper) and cow-milk-derived exosomes (lower) were analyzed using a Zeta-sizer Nano-series instrument (Malvern Nano-ZS, Malvern, UK). (C) Yak- and cow-milk-derived exosomes were quantified by Western blot, which demonstrated the enrichment of proteins of the endosomal sorting complexes required for transport (TSG101), proteins of the tetraspanin family (CD-63), heat shock protein (Hsp)-70, calnexin, and $\beta$-actin. (D) Densitometric analysis of signals obtained from exosome marker immunoblots. Data are presented as the mean \pm SEM ( $\mathrm{n}=3$ per group). Means marked with asterisks are significantly different $(* * * P<0.001)$. C-Exo $=$ cow-milk-derived exosomes added at $240 \mathrm{ng} / \mu \mathrm{L}$. Y-Exo = yak-milk-derived exosomes added at $240 \mathrm{ng} / \mu \mathrm{L}$.

method 1 for the efficient purification of milk-derived exosomes.

We isolated milk-derived exosomes from different sources (yak and cow milk; Figures 1 and 2). The purified exosomes were examined by TEM, which showed typical round particles ranging from 50 to $200 \mathrm{~nm}$ in diameter (Figure 1A). Yak-milk-derived exosomes (with a peaking diameter of $131.1 \pm 53.25 \mathrm{~nm}$ ) had a similar size distribution to that of cow milk (with a peaking diameter of $131.5 \pm 52.39 \mathrm{~nm}$; Figure 1B). We further characterized the milk-derived exosomes by Western blot to analyze their surface markers, including TSG101, CD63, Hsp70, and the absence of ER marker calnexin (Figure $1 \mathrm{C}$ and D). The results showed that yak- and cow-milk-derived exosomes produce signals for TSG101, CD63, and Hsp70 (Figure 1C and D). However, TSG101, CD63, and Hsp70 protein expression was significantly higher for yak-milk-derived exosomes than for cow-milk-derived exosomes (Figure $1 \mathrm{C}$ and D). Flow cytometry analysis showed more number of exosomes in yak milk (3.7 times compared with cow milk; $P<0.001$ ) than in cow milk (Figure $2 \mathrm{C}$ ).

\section{Experiment 2: Yak- and Cow-Milk-Derived Exosomes Promote IEC-6 Cell Proliferation in Hypoxia}

We evaluated the viability of IEC-6 cells treated with various concentrations of yak- and cow-milk-derived exosomes $(0,40,80,120,160,200$, and $240 \mathrm{ng} / \mu \mathrm{L})$ under hypoxic conditions for 6,12 , and $24 \mathrm{~h}$. The dose response data of the yak- and cow-milk-derived exosomes on IEC-6 cell survival in hypoxia are presented 
in Supplemental Figure S3 (https://doi.org/10.3168/ jds.2018-14946). The number of IEC-6 cells decreased significantly under the hypoxic conditions (negative control group; Supplemental Figure S3). Compared with cow-milk-derived exosomes, yak-milk-derived exosomes had a more efficient effect on IEC-6 cell growth under hypoxic conditions for $6 \mathrm{~h}$ but no significant differences (Supplemental Figure S3). However, compared with cow-milk-derived exosomes, yak-milk-derived exosomes exhibited significant increases in IEC-6 cell growth at $120,160,200$, and $240 \mathrm{ng} / \mu \mathrm{L}$ when under hypoxic conditions for 12 and $24 \mathrm{~h}(P<0.01$; Supplemental Figure S3). As revealed with MTT analysis, at concentration of $240 \mathrm{ng} / \mu \mathrm{L}$, yak-milk-derived exosomes significantly increased IEC-6 cell survival (rates up to 29\% under hypoxic conditions for $12 \mathrm{~h}$ ) when compared with cowmilk-derived exosomes posttreatment (rates up to $22 \%$ under hypoxic conditions for $12 \mathrm{~h} ; P<0.01$; Figure
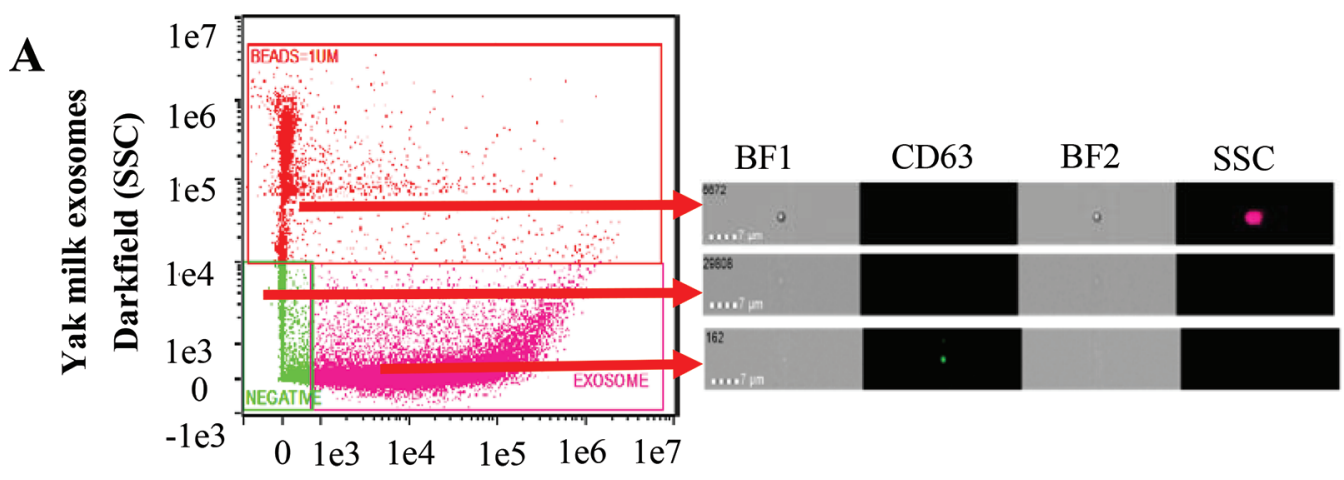

B

Fluorescence intensity - CD63

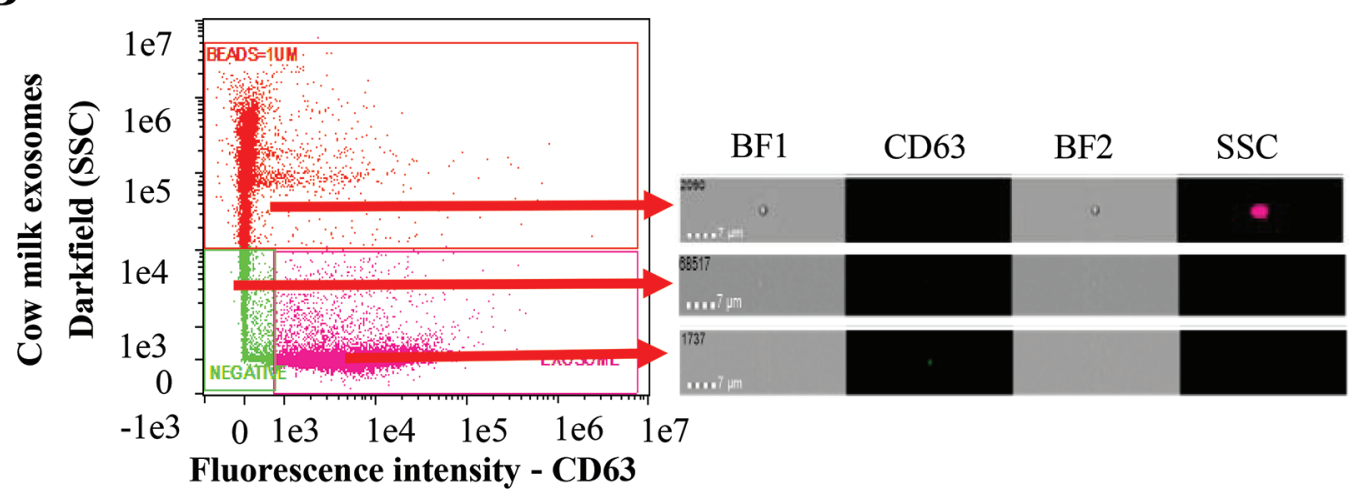

C

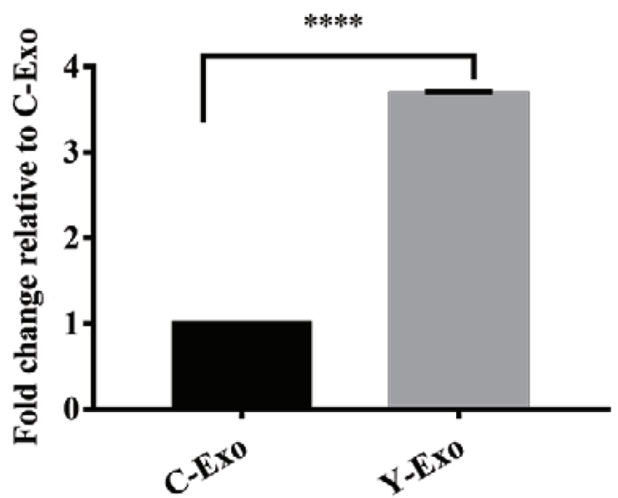

Figure 2. The quantity and size of yak- and cow-milk-derived exosomes were assessed by labeling with proteins of the tetraspanin family (CD63) and analyzed by Image Stream-X Mk II imaging cytometer (Amnis Corporation, Seattle, WA). (A) Images showing yak-milk-derived exosomes. (B) Images showing cow-milk-derived exosomes. (C) The relative number of exosomes from different milk. Data are presented as the mean \pm SEM ( $n=3$ per group). Means marked with asterisks are significantly different $(* * * * P<0.0001)$. C-Exo $=$ cow-milk-derived exosomes; Y-Exo $=$ yak-milk-derived exosomes. $\mathrm{SSC}=$ side scatter; $\mathrm{BF} 1=$ bright-field $1 ; \mathrm{BF} 2=$ bright-field 2. 
$3)$. The highest recovered number of viable IEC-6 cells was found at a concentration of $240 \mathrm{ng} / \mu \mathrm{L}$ for yakmilk-derived exosomes under hypoxic conditions for 12 h $(P<0.01$; Figure 3$)$. Using a proliferation recovery of $\geq 20 \%$ as a criterion, compared with the negative control (all of these responses were maximized), we selected the concentration of $240 \mathrm{ng} / \mu \mathrm{L}$ for yak- and cow-milk-derived exosomes to treat the IEC-6 cells for $12 \mathrm{~h}$ in the subsequent experiments.

\section{Experiment 3: Uptake of Milk-Derived Exosomes by IEC-6 Cells in Hypoxia}

The confocal microscopy revealed that the yak- and cow-milk-derived exosomes were taken up by the IEC-6 cells in both normoxic and hypoxic conditions for $12 \mathrm{~h}$ (Figure 4). However, exosome incorporation was significantly higher in normoxic than in hypoxic conditions (Figure 4); IEC-6 cells uptake significantly more yak-
A

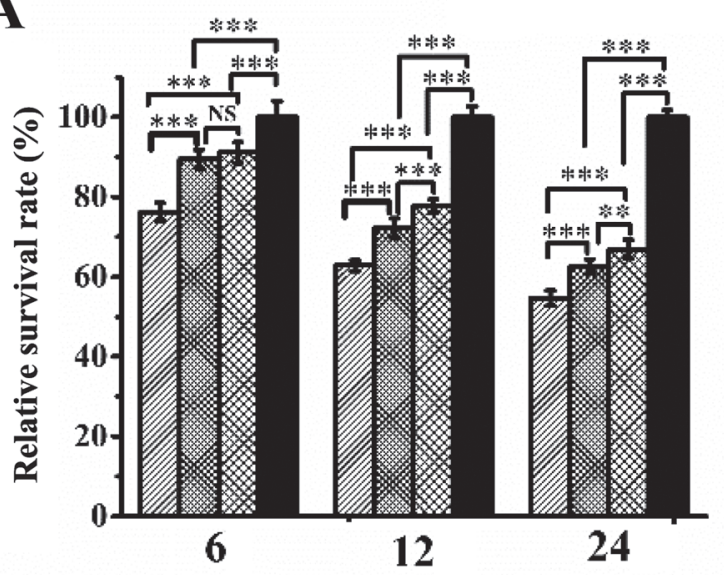

$\mathbf{C}$

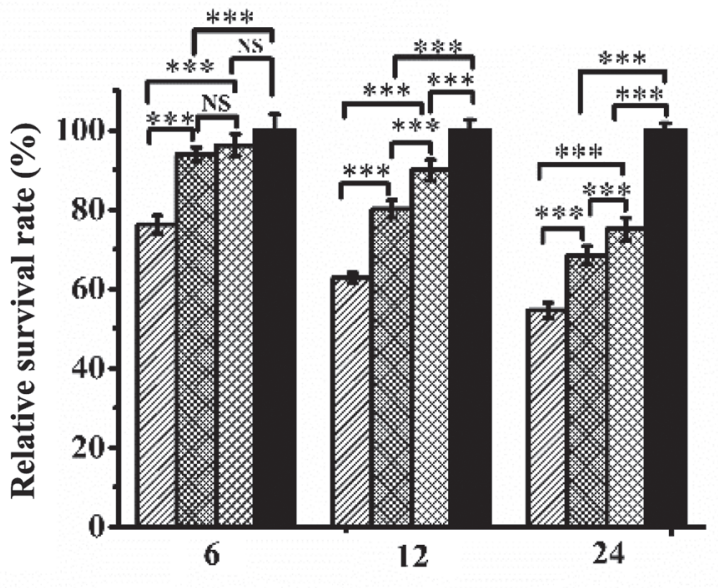

Time (h)
B

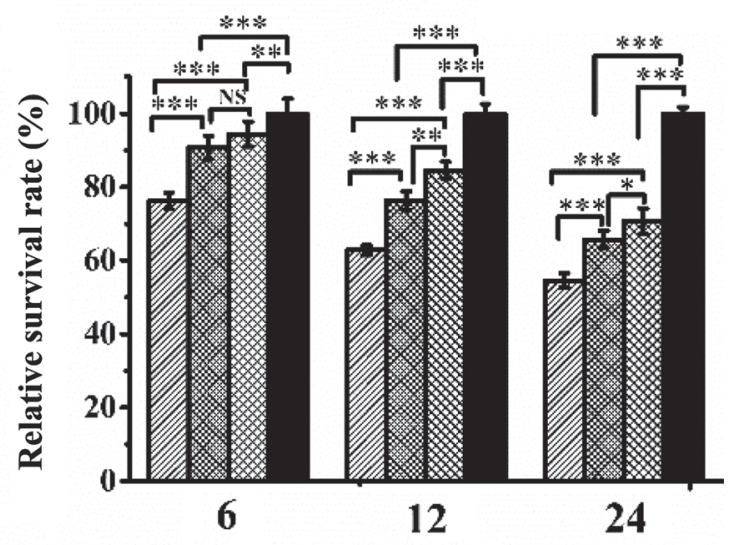

D

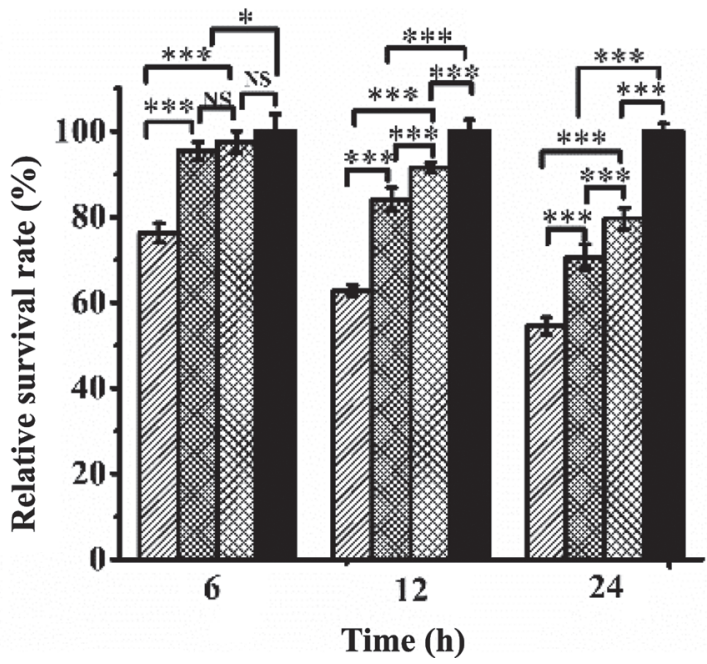

Figure 3. Effect of milk-derived (yak and cow) exosomes (EXO) on the relative survival rate (\%) of intestinal epithelial-6 cells (IEC-6) under hypoxic conditions. (A) The IEC-6 cells were cultured with the absence or presence of $120 \mathrm{ng} / \mu \mathrm{L}$ of yak- and cow-milk-derived exosomes under hypoxic conditions for various periods of time. (B) The IEC- 6 cells were cultured with the absence or presence of $160 \mathrm{ng} / \mu \mathrm{L}$ of yak- and cow-milk-derived exosomes under hypoxic conditions for various periods of time. (C) The IEC-6 cells were cultured with the absence or presence of $200 \mathrm{ng} / \mu \mathrm{L}$ of yak- and cow-milk-derived exosomes under hypoxic conditions for various periods of time. (D) The IEC-6 cells were cultured with the absence or presence of $240 \mathrm{ng} / \mu \mathrm{L}$ of yak- and cow-milk-derived exosomes under hypoxic conditions for various periods of time. Data are expressed as the percentage of the supplemented yak and cow milk exosomes in hypoxic relative to normoxia control, in the form of means \pm SEM ( $\mathrm{n}=6$ per group). Means marked with asterisks are significantly different $\left(* P<0.05 ;{ }^{* *} P<0.01 ; * * * P<0.001\right)$. Normoxia control: IEC-6 cells in normoxia conditions (absence of yak- and cow-milk-derived exosomes). 


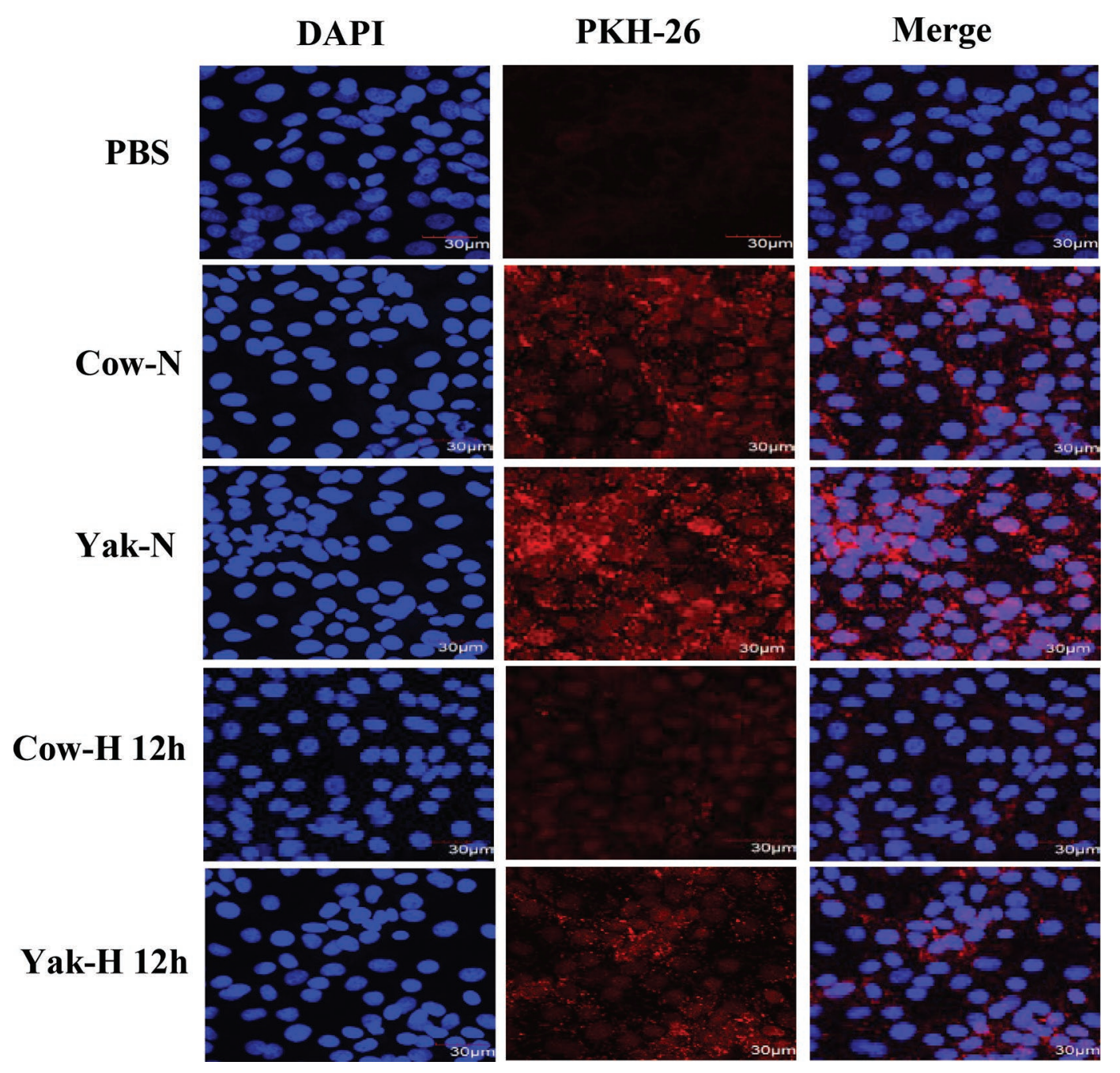

Figure 4. Uptake of yak- and cow-milk-derived exosomes by intestinal epithelial-6 cells (IEC-6). Confocal microscopy images showing IEC-6 nuclei labeled with 4',6'-diaminido-2-phenylindole (DAPI, blue) and exosomes labeled with PKH26 dye (red). PBS: PBS-PKH26 was added to the IEC-6 cells, followed by incubation at $5 \% \mathrm{CO}_{2}$ for $12 \mathrm{~h}$ (control). Yak-N and Cow-N: PKH26-labeled yak and cow exosomes were added to the IEC-6 cells, followed by incubation at $5 \% \mathrm{CO}_{2}$ for $12 \mathrm{~h}$ (normoxic). Yak-H and Cow-H: PKH26-labeled yak and cow milk exosomes were added to the IEC- 6 cells at $5 \% \mathrm{CO}_{2}, 95 \% \mathrm{~N}_{2}$ for $12 \mathrm{~h}$ (hypoxic).

milk-derived exosomes than cow-milk-derived exosomes in hypoxic conditions (Figure 4). The extent of exosome incorporation into the IEC-6 cells was analyzed using confocal microscopy (Figure 4).

\section{Experiment 4: Effect of IEC-6 Cells in Hypoxia on HIF and p53-Related Protein Expression}

To further explore the effects of yak- and cow-milkderived exosomes on intestinal tract development under hypoxic conditions, HIF signaling pathway-related and proliferation-related protein expression was analyzed. The p53 protein levels were increased in the IEC-6 cells under hypoxic conditions (Figure 5A-B). The p53 expression was decreased after IEC-6 cells were treated with $240 \mathrm{ng} / \mu \mathrm{L}$ yak- and cow-milk-derived exosomes, compared with that before treatment. The results showed significantly inhibited p53 levels in IEC-6 cells after incubation with yak-milk-derived exosomes (Figure $5 \mathrm{~A}-\mathrm{B}$ ) relative to that after incubation with cowmilk-derived exosomes. The yak-milk-derived exosomes were able to regulate the anti-hypoxia capacity of the IEC-6 cells to an even greater extent than the cowmilk-derived exosomes.

The results showed that HIF- $\alpha$ and VEGF proteins were highly expressed in hypoxic conditions (Figure $5 \mathrm{C}-\mathrm{D})$. However, PHD-1 showed low expression in hypoxic conditions. Compared with the negative group, 
yak-milk-derived exosomes significantly promoted the expression of PHD-1 and decreased the expression of HIF- $\alpha$ and its downstream target VEGF in the IEC6 cells (Figure 5C-D). Interestingly, HIF- $\alpha$ expression was similar to that of p53 expression in the IEC-6 cells under hypoxic conditions (Figure 5). The Western blot analysis further confirmed that yak-milk-derived exosomes more effectively activate the HIF signaling pathway, thus promoting IEC-6 cells survival in hypoxia, than cow-milk-derived exosomes.

\section{DISCUSSION}

Recent studies have revealed milk exosomes to be small (30-200 nm) membrane-derived extracellular vesicles that carry a wide range of functional proteins, mRNA, and microRNA and mediate intercellular communication (Wolf et al., 2015; Kusuma et al., 2016). Research has showed that milk-derived exosomes can promote IEC cell proliferation (Chen et al., 2016; Hock et al., 2017). Some reports indicated that altitude-
A

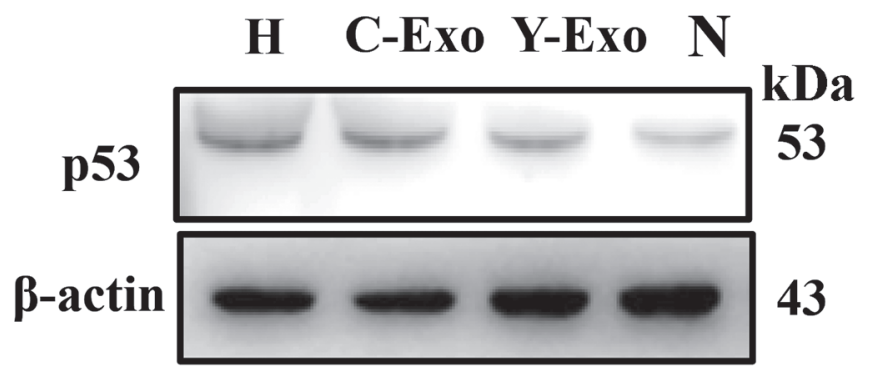

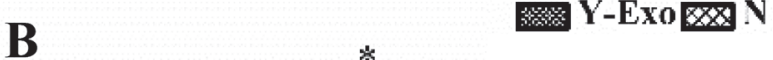

H Ema C-Exo

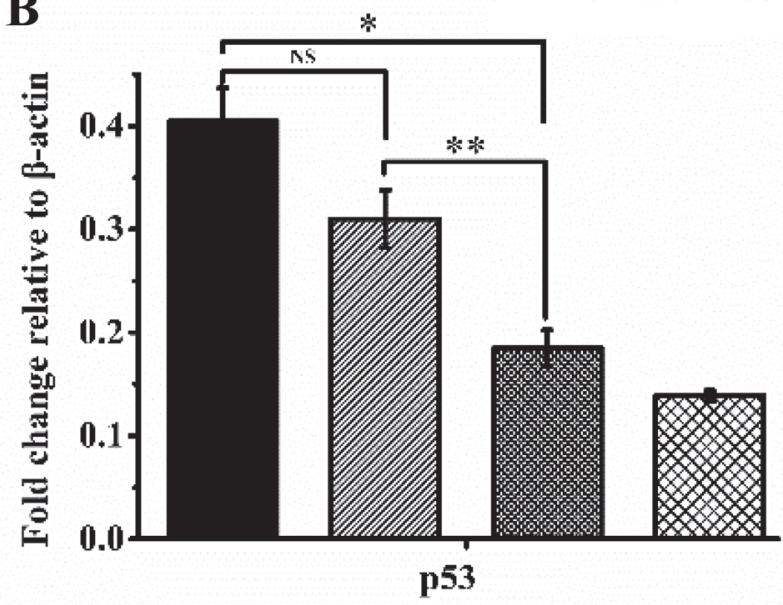

D
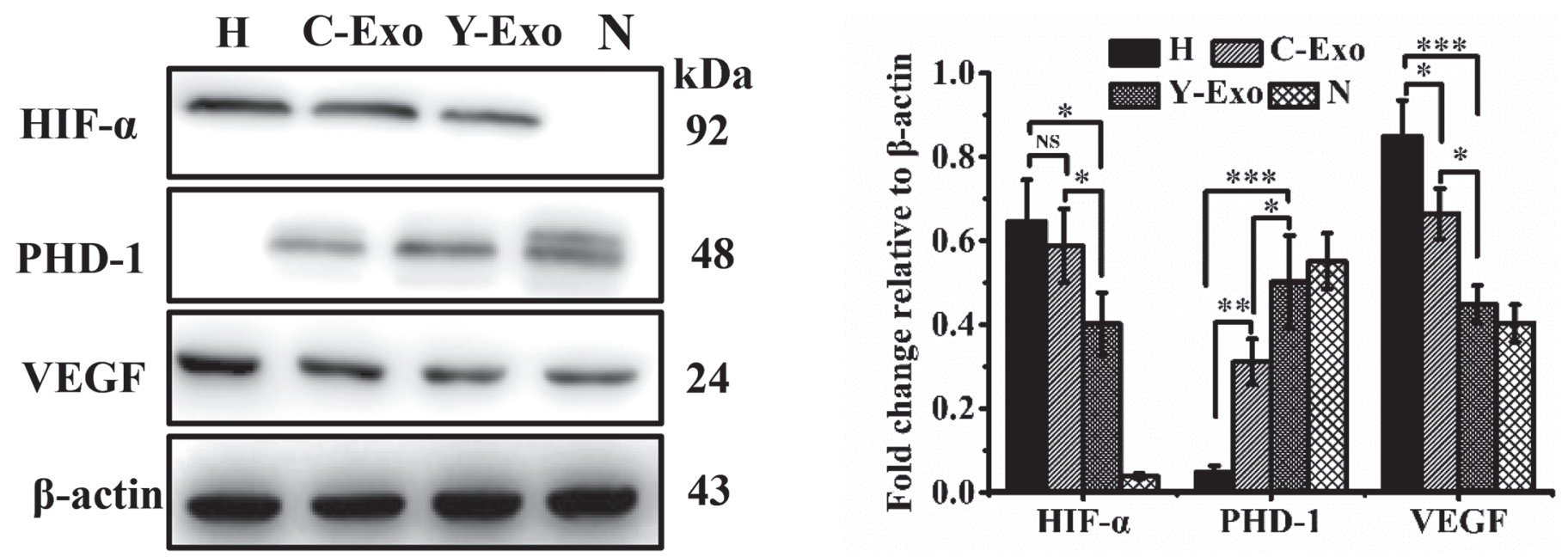

Figure 5. Effect of intestinal epithelial-6 cells (IEC-6) under hypoxic conditions on expression of p53 and hypoxia-inducible factor (HIF)related proteins. Yak- and cow-milk-derived exosomes were added under hypoxic conditions for $12 \mathrm{~h}$ at $240 \mathrm{ng} / \mu \mathrm{L}$. Representative blots of p53 and HIF-related proteins are shown in panels A and C. $\beta$-Actin was assessed as a loading control. Panels B and D indicate densitometric analysis of signals obtained from the p53 and HIF-related protein immunoblots. Data are presented as the mean \pm SEM ( $\mathrm{n}=3$ per group). Means marked with asterisks are significantly different $(* P<0.05 ; * *<0.01 ; * * * P<0.001)$. $\mathrm{H}=$ incubation under hypoxic conditions $(5 \%$ $\left.\mathrm{CO}_{2}, 95 \% \mathrm{~N}_{2}\right) . \mathrm{N}=$ incubation under normoxic conditions for $12 \mathrm{~h}\left(5 \% \mathrm{CO}_{2}\right)$. Y-Exo = yak-milk-derived exosomes added at $240 \mathrm{ng} / \mu \mathrm{L}$. C-Exo $=$ cow-milk-derived exosomes added at $240 \mathrm{ng} / \mu \mathrm{L} . \mathrm{PHD}=$ prolyl hydroxylase; VEGF = vascular endothelial growth factor. 
associated hypoxia could damage gut barrier function in the duodenum of healthy individuals (Vavricka et al., 2014; Wojtal et al., 2014; Vavricka et al., 2016). Cell renewal is key to maintaining the epithelial barrier (Manresa and Taylor, 2017), and controlling epithelial cell renewal is a continuous balance between proliferation and apoptosis-dependent cell death (Ramachandran et al., 2000; Gregorieff and Clevers, 2005; van der Flier and Clevers, 2009). However, the functions of yak-milkderived exosomes in the intestinal tissues under hypoxic conditions have still not been fully characterized.

The isolation of exosomes from milk is currently a nonspecific and difficult process. We describe here a novel approach (ultracentrifugation combined with rennet) with the aim to increase the efficiency of isolating exosomes from cow milk, compared with previous protocols, which only used ultracentrifugation. The samples obtained using our method contained round structures with a peaking diameter of 50 to $200 \mathrm{~nm}$, which is concordant with previous reports (Théry et al., 2006; Li et al., 2016).

We show here the presence of exosomes in yak and cow milk that display all the hallmarks of exosomes (Clayton et al., 2001; Théry et al., 2006; Li et al., 2016): (1) shape and size 50 to $200 \mathrm{~nm}$, and (2) expression of different exosome-associated molecules on the surface, such as TSG101, CD63, and Hsp70, and the lack of the ER-specific marker calnexin. Compared with cow-milkderived exosomes, yak-milk-derived exosomes showed significantly higher expression of the proteins TSG101, CD63, and Hsp70 as determined by Western blot. Further, significantly more exosomes were found in yak milk than in cow milk $(P<0.001)$ analyzed by flow cytometry.

In this study, we studied the effect of yak-milk-derived exosomes on the HIF- $\alpha$ signaling pathway in the regulation of GIT development under hypoxic conditions. Our findings are consistent with those of previous research demonstrating that the number of cells decreased significantly under hypoxic conditions (Kucharzewska et al., 2013). Milk exosomes are biologically active vesicles, and a previous study strongly suggested that milk-derived exosomes are able to promote intestinal cell proliferation and digestive tract development (Chen et al., 2016). Cell proliferation is the primary driver of intestinal growth and development (Reznikov et al., 2014). In agreement with previous studies, our results suggest an increase in IEC-6 proliferation induced by yak- and cow-milk-derived exosomes. Compared with cow-milk-derived exosomes, yak-milkderived exosomes had a more efficient effect on IEC-6 cell growth under hypoxic conditions. Under hypoxic conditions, when the concentration of exosomes was at 200 to $240 \mathrm{ng} / \mu \mathrm{L}$, the yak-milk-derived exosomes increased IEC-6 cell survival significantly more than the cow-milk-derived exosomes posttreatment (rates up to 29 vs. $22 \%$, respectively; $P<0.01$ ). Here, we provide evidence suggesting that specific components of the yak milk exosomes could potentially improve this hypoxia resistance in IEC-6 cells.

Our study provides compelling evidence that IEC-6 cells can take up yak- and cow-milk-derived exosomes by an active process that is yet to be determined. Consistent with this, some studies indicate that in vitro, the murine small intestine and intestinal cells can take up bovine-milk-derived extracellular vesicles by endocytosis (Arntz et al., 2015; Wolf et al., 2015); further, human macrophages can take up exosomes from human breast and cow milk by an active process (Lässer et al., 2011; Izumi et al., 2015, respectively); human vascular endothelial cells can take up cow milk exosomes via endocytosis (Kusuma et al., 2016). The above research show that milk-derived exosomes may cross the intestinal mucosa and have the ability to biologically activate the response signal. To the best of our knowledge, this is the first paper to propose that, under hypoxic conditions, the transport of yak- and cow-milk-derived exosomes across IEC-6 cells is mediated by endocytosis. However, we showed that yak-milk-derived exosomes are taken up by IEC- 6 cells to a greater extent than cow-milk-derived exosomes under hypoxic conditions. We suspect that some specific components in yak milk exosomes can more effectively alleviate the effects of hypoxia and promote the absorption of small molecules.

To further explore how yak-milk-derived exosomes can affect IEC-6 cell survival, we studied the expression levels of proteins related to intestinal cell survival and the HIF- $\alpha$ signaling pathway. Our results show that hypoxic environments can increase HIF- $\alpha$ and VEGF expression concomitantly with the induction of p53 protein expression in IEC- 6 cells. The p53 protein is the main negative regulator of the cell cycle. A rapid rise in p53 levels can inhibit cell growth, and tight regulation of p53 function is critical for normal cell growth and development (Kubbutat et al., 1997; Chen et al., 2016). Therefore, compared with the negative group, p53 expression decreased after the IEC-6 cells were treated with $240 \mathrm{ng} / \mu \mathrm{L}$ of yak- and cow-milk-derived exosomes, and as demonstrated in previous studies, p53 expression is a determining factor for cell survival (Afanasyeva et al., 2011; Chen et al., 2016). However, yak-milk-derived exosomes more strongly inhibit p53 expression than cow-milk-derived exosomes, which is consistent with our MTT assay results.

Evidence is mounting that HIF- $\alpha$ expression is increased in the inflamed mucosa of patients with inflammatory bowel disease, which is regulated by various inflammatory stimuli (Colgan and Taylor, 2010; Manresa 
and Taylor, 2017; Brown and Taylor, 2018). Hypoxia leads to the inactivation of PHD enzymes, which in turn leads to the stabilization of HIF (Manresa and Taylor, 2017; Brown and Taylor, 2018) Our results indicate that the HIF- $\alpha$ and VEGF proteins are highly expressed in hypoxia. Our results indicate that the addition of yak- and cow-milk-derived exosomes can increase PHD-1 expression and decrease the expression of HIF- $\alpha$ and its downstream target VEGF, in comparison with the IEC-6 cell hypoxia group. Our results confirmed stronger activation of the HIF signaling pathway promoting IEC-6 cell survival under hypoxia by yak-milk-derived exosomes than cow-milk-derived exosomes.

\section{CONCLUSIONS}

We describe here a novel approach (ultracentrifugation combined with rennet) with the aim to increase the efficiency of isolating exosomes from milk. Our results showed that compared with previous protocols, our protocol is more suitable for the efficient purification of milk-derived exosomes. The results of the present study indicate that compared with cow-milk-derived exosomes, yak-milk-derived exosomes had a more efficient effect on IEC-6 cell growth under hypoxic conditions. We found that IEC-6 cells mixed with $240 \mathrm{ng} / \mu \mathrm{L}$ of yak-milk-derived exosomes had significantly higher cell survival rates of up to $29 \%$ when under hypoxic conditions for $12 \mathrm{~h}$ than cow-milk-derived exosomes posttreatment (rates of up to $22 \%$ when under hypoxic conditions for $12 \mathrm{~h}$ ). These findings will help to improve yak-milk-derived exosome regulation of GIT development under hypoxic conditions. However, more research is required to translate these findings to a long-term in vivo model.

\section{ACKNOWLEDGMENTS}

This work was partially funded by The 111 project from the Education Ministry of China (No. B18053) and National Key R\&D Program of China (No. 2017YFD0400605), the FUXI young talent (Gaufx02Y01). All authors read and approved the final manuscript.

\section{REFERENCES}

Afanasyeva, E. A., P. Mestdagh, C. Kumps, J. Vandesompele, V. Ehemann, J. Theissen, M. Fischer, M. Zapatka, B. Brors, and L. Savelyeva. 2011. MicroRNA miR-885-5p targets CDK2 and MCM5, activates p53 and inhibits proliferation and survival. Cell Death Differ. 18:974-984.

Al-Nedawi, K., B. Meehan, and J. Rak. 2009. Microvesicles: Messengers and mediators of tumor progression. Cell Cycle 8:2014-2018.
Arntz, O. J., B. C. Pieters, M. C. Oliveira, M. G. Broeren, M. B. Bennink, V. M. De, P. L. van Lent, M. I. Koenders, V. D. B. Wb, and V. D. K. Pm. 2015. Oral administration of bovine milk-derived extracellular vesicles attenuates arthritis in two mouse models. Mol. Nutr. Food Res. 59:1701-1712.

Aro, E., R. Khatri, R. Gerardo-Riley, L. Mangiavini, J. Myllyharju, and E. Schipani. 2012. Hypoxia-inducible factor-1 (HIF-1) but not HIF-2 is essential for hypoxic induction of collagen prolyl 4-hydroxylases in primary newborn mouse epiphyseal growth plate chondrocytes. J. Biol. Chem. 287:37134-37144.

Belting, M., and A. Wittrup. 2008. Nanotubes, exosomes, and nucleic acid-binding peptides provide novel mechanisms of intercellular communication in eukaryotic cells: Implications in health and disease. J. Cell Biol. 183:1187-1191.

Benmoussa, A., C. H. Lee, B. Laffont, P. Savard, J. Laugier, E. Boilard, C. Gilbert, I. Fliss, and P. Provost. 2016. Commercial dairy cow milk microRNAs resist digestion under simulated gastrointestinal tract conditions. J. Nutr. 146:2206-2215.

Brown, E., and C. T. Taylor. 2018. Hypoxia-sensitive pathways in intestinal inflammation. J. Physiol. 596:2985-2989.

Chen, T., M. Y. Xie, J. J. Sun, R. S. Ye, X. Cheng, R. P. Sun, L. M. Wei, M. Li, D. L. Lin, and Q. Y. Jiang. 2016. Porcine milk-derived exosomes promote proliferation of intestinal epithelial cells. Sci. Rep. 6:33862.

Chen, X., C. Gao, H. Li, L. Huang, Q. Sun, Y. Dong, C. Tian, S. Gao, H. Dong, and D. Guan. 2010. Identification and characterization of microRNAs in raw milk during different periods of lactation, commercial fluid, and powdered milk products. Cell Res. 20:1128-1137.

Clayton, A., J. Court, H. Navabi, M. Adams, M. D. Mason, J. A. Hobot, G. R. Newman, and B. Jasani. 2001. Analysis of antigen presenting cell derived exosomes, based on immuno-magnetic isolation and flow cytometry. J. Immunol. Methods 247:163-174.

Cocucci, E., G. Racchetti, and J. Meldolesi. 2009. Shedding microvesicles: Artefacts no more. Trends Cell Biol. 19:43-51.

Colgan, S. P., and C. T. Taylor. 2010. Hypoxia: An alarm signal during intestinal inflammation. Nat. Rev. Gastroenterol. Hepatol. $7: 281-287$.

Gregorieff, A., and H. Clevers. 2005. Wnt signaling in the intestinal epithelium: From endoderm to cancer. Genes Dev. 19:877-890.

Gu, Y., M. Li, T. Wang, Y. Liang, Z. Zhong, X. Wang, Q. Zhou, L. Chen, Q. Lang, and Z. He. 2012. Lactation-related microRNA expression profiles of porcine breast milk exosomes. PLoS One $7: \mathrm{e} 43691$.

Hata, T., K. Murakami, H. Nakatani, Y. Yamamoto, T. Matsuda, and N. Aoki. 2010. Isolation of bovine milk-derived microvesicles carrying mRNAs and microRNAs. Biochem. Biophys. Res. Commun. 396:528-533.

Hock, A., H. Miyake, B. Li, C. Lee, L. Ermini, Y. Koike, Y. Chen, P. Määttänen, A. Zani, and A. Pierro. 2017. Breast milk-derived exosomes promote intestinal epithelial cell growth. J. Pediatr. Surg. 52:755-759

Izumi, H., N. Kosaka, T. Shimizu, K. Sekine, T. Ochiya, and M. Takase. 2012. Bovine milk contains microRNA and messenger RNA that are stable under degradative conditions. J. Dairy Sci. 95:4831-4841.

Izumi, H., M. Tsuda, Y. Sato, N. Kosaka, T. Ochiya, H. Iwamoto, K. Namba, and Y. Takeda. 2015. Bovine milk exosomes contain microRNA and mRNA and are taken up by human macrophages. J. Dairy Sci. 98:2920-2933.

Jiang, G., R. Zhou, X. He, Z. Shi, M. Huang, J. Yu, and X. Wang. 2016. Expression levels of microRNA-199 and hypoxia-inducible factor-1 alpha in brain tissue of patients with intractable epilepsy. Int. J. Neurosci. 126:326-334.

Karhausen, J., G. T. Furuta, J. E. Tomaszewski, R. S. Johnson, S. P. Colgan, and V. H. Haase. 2004. Epithelial hypoxia-inducible factor- 1 is protective in murine experimental colitis. J. Clin. Invest. 114:1098-1106.

Kosaka, N., H. Izumi, K. Sekine, and T. Ochiya. 2010. microRNA as a new immune-regulatory agent in breast milk. Silence 1:7.

Kubbutat, M. H., S. N. Jones, and K. H. Vousden. 1997. Regulation of p53 stability by Mdm2. Nature 387:299-303. 
Kucharzewska, P., H. C. Christianson, J. E. Welch, K. J. Svensson, E. Fredlund, M. Ringnér, M. Mörgelin, E. Bourseauguilmain, J. Bengzon, and M. Belting. 2013. Exosomes reflect the hypoxic status of glioma cells and mediate hypoxia-dependent activation of vascular cells during tumor development. Proc. Natl. Acad. Sci. USA 110:7312-7317.

Kusuma, R. J., S. Manca, T. Friemel, S. Sukreet, C. Nguyen, and J. Zempleni. 2016. Human vascular endothelial cells transport foreign exosomes from cow's milk by endocytosis. Am. J. Physiol. Cell Physiol. 310:C800-C807.

Lässer, C., V. S. Alikhani, K. Ekström, M. Eldh, P. T. Paredes, A. Bossios, M. Sjöstrand, S. Gabrielsson, J. Lötvall, and H. Valadi. 2011. Human saliva, plasma and breast milk exosomes contain RNA: Uptake by macrophages. J. Transl. Med. 9:9. https://doi .org/10.1186/1479-5876-9-9.

Li, L., C. Li, S. Wang, Z. Wang, J. Jiang, W. Wang, X. Li, J. Chen, K. Liu, and C. Li. 2016. Exosomes derived from hypoxic oral squamous cell carcinoma cells deliver miR-21 to normoxic cells to elicit a prometastatic phenotype. Cancer Res. 76:1770-1780.

Liao, Y., X. Du, J. Li, and B. Lönnerdal. 2017. Human milk exosomes and their microRNAs survive digestion in vitro and are taken up by human intestinal cells. Mol. Nutr. Food Res. 61. https://doi .org/10.1002/mnfr.201700082.

Manresa, M. C., and C. T. Taylor. 2017. Hypoxia inducible factor (HIF) hydroxylases as regulators of intestinal epithelial barrier function. Cell. Mol. Gastroenterol. Hepatol. 3:303-315.

Munch, E. M., H. R. Alan, M. Mahmoud, A. L. Benham, S. M. Pejerrey, S. Lori, H. Min, C. D. Shope, P. D. Maningat, and P. H. Gunaratne. 2013. Transcriptome profiling of microRNA by Next-Gen deep sequencing reveals known and novel miRNA species in the lipid fraction of human breast milk. PLoS One 8:e50564.

Nivsarkar, A. E., S. C. Gupta, N. Gupta, A. E. Nivsarkar, S. C. Gupta, and N. Gupta. 1997. Yak Production. ICAR Publication, New Delhi, India.

Qiu, Q., G. Zhang, T. Ma, W. Qian, J. Wang, Z. Ye, C. Cao, Q. Hu, J. Kim, and D. M. Larkin. 2012. The yak genome and adaptation to life at high altitude. Nat. Genet. 44:946-949.

Ramachandran, A., M. Madesh, and K. A. Balasubramanian. 2000 Apoptosis in the intestinal epithelium: Its relevance in normal and pathophysiological conditions. J. Gastroenterol. Hepatol. 15:109120.

Reznikov, E. A., S. S. Comstock, C. Yi, N. Contractor, and S. M. Donovan. 2014. Dietary bovine lactoferrin increases intestinal cell proliferation in neonatal piglets. J. Nutr. 144:1401-1408.

Sun, M., C. He, W. Wu, G. Zhou, F. Liu, Y. Cong, and Z. Liu. 2017. Hypoxia inducible factor- $1 \alpha$-induced IL-33 expression in intestinal epithelia contributes to mucosal homeostasis in inflammatory bowel disease. Clin. Exp. Immunol. 187:428-440.
Sun, Q., X. Chen, J. Yu, K. Zen, C. Y. Zhang, and L. Li. 2013. Immune modulatory function of abundant immune-related microRNAs in microvesicles from bovine colostrum. Protein Cell 4:197-210.

Taylor, C. T., and S. P. Colgan. 2007. Hypoxia and gastrointestinal disease. J. Mol. Med. 85:1295-1300.

Théry, C., S. Amigorena, G. Raposo, and A. Clayton. 2006. Isolation and Characterization of Exosomes from Cell Culture Supernatants and Biological Fluids. John Wiley \& Sons Inc., Hoboken, NJ.

Théry, C., M. Ostrowski, and E. Segura. 2009. Membrane vesicles as conveyors of immune responses. Nat. Rev. Immunol. 9:581-593.

van der Flier, L. G., and H. Clevers. 2009. Stem cells, self-renewal, and differentiation in the intestinal epithelium. Annu. Rev. Physiol. 71:241-260.

Vavricka, S. R., G. Rogler, and L. Biedermann. 2016. High altitude journeys, flights and hypoxia: Any role for disease flares in IBD patients? Dig. Dis. 34:78-83.

Vavricka, S. R., G. Rogler, S. Maetzler, B. Misselwitz, E. Safroneeva, P. Frei, C. N. Manser, L. Biedermann, M. Fried, and P. Higgins. 2014. High altitude journeys and flights are associated with an increased risk of flares in inflammatory bowel disease patients. J. Crohn's Colitis 8:191-199.

Wojtal, K. A., A. Cee, S. Lang, O. Götze, H. Frühauf, A. Geier, M. Pastor-Anglada, J. Torres-Torronteras, R. Martí, and M. Fried. 2014. Downregulation of duodenal SLC transporters and activation of proinflammatory signaling constitute the early response to high altitude in humans. Am. J. Physiol. Gastrointest. Liver Physiol. 307:G673-G688.

Wolf, T., S. R. Baier, and J. Zempleni. 2015. The intestinal transport of bovine milk exosomes is mediated by endocytosis in human colon carcinoma Caco-2 cells and rat small intestinal IEC-6 cells. J. Nutr. 145:2201-2206.

Yang, M., D. Song, X. Cao, R. Wu, B. Liu, W. Ye, J. Wu, and X. Yue 2017. Comparative proteomic analysis of milk-derived exosomes in human and bovine colostrum and mature milk samples by iTRAQcoupled LC-MS/MS. Food Res. Int. 92:17-25.

Yang, Y., N. Zheng, J. Yang, D. Bu, J. Wang, L. Ma, and P. Sun 2014. Animal species milk identification by comparison of twodimensional gel map profile and mass spectrometry approach. Int. Dairy J. 35:15-20.

Zaborowski, M. P., L. Balaj, X. O. Breakefield, and C. P. Lai. 2015 Extracellular vesicles: Composition, biological relevance, and methods of study. Bioscience 65:783-797.

Zhang, Y., Y. Li, P. Wang, Y. Tian, Q. Liang, and F. Ren. 2017. Rennet-induced coagulation properties of yak casein micelles: A comparison with cow casein micelles. Food Res. Int. 102:25-31.

Zhou, Q., M. Li, X. Wang, Q. Li, T. Wang, Q. Zhu, X. Zhou, X. Wang, X. Gao, and X. Li. 2012. Immune-related microRNAs are abundant in breast milk exosomes. Int. J. Biol. Sci. 8:118-123. 